\title{
Papers
}

\section{Treatment classification trees: A method for offer segmentation in direct marketing}

\author{
RECEIVED (IN REVISED FORM): 4 SEPTEMBER, 2000 \\ Penny N. Spring \\ Department of Economics, University of Groningen, PO Box 800, 9700 AV Groningen, \\ The Netherlands. \\ Tel: + I 050363 7320; Fax: + I 050363 3720; e-mail: p.n.spring@eco.rug.nl
}

Penny N. Spring

is a doctoral candidate at the

Department of

Economics at the

University of

Groningen in the

Netherlands. She

holds a BA in

Mathematics from

Wells College, and

an MS in

Operations

Research and

Statistics from

Rensselaer

Polytechnic

Institute. She has

worked in direct

marketing in both

the USA and

Europe. Her

research interests

include the

application of

statistics to

optimise direct

marketing efforts.

\section{Abstract}

Direct mail offer designers and copywriters strive to create the offer which will yield the greatest response for a particular product offering. Testing is generally performed to determine which offer of the many candidates has the broadest appeal. Once the test results are compiled, usually a single offer will be chosen and sent to all targets in a bulk mailing. The assumption made here is that all the targets will prefer the offer which was deemed best in the tests. The hypothesis is that consumers are not homogenous in their reactions to offers, and that customers' characteristics can be used to determine which offer is best on an individual, not a list basis. This paper proposes a technique for classifying consumers into offer groups based on test samples of different offers. This new technique is called Treatment Classification Trees, or TaCT. The first application shown illustrates when this method is preferred over a standard logit model. Also presented is a case study using actual test data showing that profits can be increased using TaCT.

\section{INTRODUCTION}

An important task of direct marketers is to determine the best offer for a certain mailing. The offer is comprised of a myriad of elements such as the product, price, brochure, letter, premium, envelopes, endorsements, etc. The decision as to precisely which elements should make up the offer is generally made for the customer population as a whole. Thus, the question direct marketers ask themselves is which single combination of offer elements will generate the greatest profits in a particular mailing for my mailing list?

Testing plays a great role in determining which offer is optimal. Small random samples of customers are taken from the customer population. The consumers in each sample receive one of the candidate offers. The direct marketer subsequently tallies the responses to each of the candidate offers. If one offer generates significantly higher profits than the others, it will be chosen for the mailing 
to the customer population in the rollout mailing.

In this situation all selected prospects in the rollout will receive the offer deemed most profitable for the whole list. This strategy is potentially wasteful because it assumes that a single offer will be the most appealing to everyone on the list. The contention in this paper is that an offer which is relatively less appealing to the whole list will be the most enticing to some people. Further, if the marketer has conducted multiple offer tests and has demographic or past purchase data for each customer, then it is possible to use those data to identify customer characteristics which indicate preference for one type of offer or the other.

The purpose of this paper is to propose and demonstrate a method for segmenting customers into offer groups based on their characteristics and their responses to offer tests. This new method is called Treatment Classification Trees, or TaCT. There are two previous studies in the literature which address data of the kind described here. The first, by Bult, van der Scheer and Wansbeek analyses tests of varying offers with a logit model which includes interaction variables with offer indicators. ${ }^{1}$ Their paper stressed design aspects. The same modelling method is utilised by Spring, Leeflang and Wansbeek, ${ }^{2}$ but here a segmentation strategy for offer assignment is proposed.

This paper, first discusses the inspection of odds ratios as a method for comparing proportions. This is because odds ratios are convenient for comparing the response percentages of groups of customers given different offers. The paper continues with a proposal for a tree-based technique for segmenting customers into offer groups based on odds ratios. The algorithm is very similar to the Classification and Regression Trees technique (CART) and follows the outline proposed by Breiman et al., ${ }^{3}$ who developed CART. Two examples are shown. The first illustrates a case where the new method operates more simply than a standard logit model. A case study is also presented, showing results from two DM offer tests. The paper concludes with a section discussing business implications and future steps for this research.

\section{Odds ratios and offer segmentation}

Odds ratios are convenient statistics for comparing ratios, and are thus useful in examining order rates. Consider two direct marketing test samples, each with a differing response (or 'success') rate. The purpose is to investigate the relation between the response rates. To that end, in this section odds ratios are introduced and their properties discussed. Let us define two independent binomially distributed random variables $X_{1}$ and $X_{2}$, analogous to test samples 1 and 2, such that $X_{1} \sim B\left(\pi_{11}, n_{1}\right)$ and $X_{2} \sim B\left(\pi_{21}, n_{2}\right)$, where $\pi_{11}$ and $\pi_{21}$ represent the binomial parameters, and $n_{1}$ and $n_{2}$ are the fixed sample sizes. Let $\pi_{12}=1-\pi_{11}$ and $\pi_{22}=1-\pi_{21}$. The 
odds ratio is defined as:

$$
\theta=\frac{\pi_{11} \pi_{22}}{\pi_{21} \pi_{12}}
$$

Results from a random draw from $X_{1}$ and $X_{2}$ can be summarised in a frequency table, see Table 1 . Let

$$
\begin{aligned}
n & =n_{11}+n_{12}+n_{21}+n_{22} \\
n_{1} & =n_{11}+n_{12} \\
n_{2} & =n_{21}+n_{22} \\
\hat{\pi}_{11} & =\frac{n_{11}}{n_{1}} \\
\hat{\pi}_{21} & =\frac{n_{21}}{n_{2}} \\
\hat{\pi}_{12} & =1-\hat{\pi}_{11} \\
\hat{\pi}_{22} & =1-\hat{\pi}_{21}
\end{aligned}
$$

The observed odds that an observation in Sample 1 is a 1-responder is $n_{11} / n_{12}$. Likewise, the observed odds that an observation in Sample 2 is a 1 -responder is $n_{21} / n_{22}$. The observed odds ratio between the two samples is then:

$$
\hat{\boldsymbol{\theta}}=\frac{n_{11} n_{22}}{n_{21} n_{12}}=\frac{\hat{\pi}_{11} \hat{\pi}_{22}}{\hat{\pi}_{21} \hat{\pi}_{12}} .
$$

This statistic is also known as the cross-product ratio. It can take on values of 0 or greater. If a cell is empty, the value can be infinite. A value of 1 indicates that an observation in Sample 1 is equally likely to be a 1 -responder as an observation from Sample 2. A value of 5 indicates that the odds of a 1-response is five times higher in Sample 1 than in Sample 2. Let

$$
\hat{\lambda}=\ln \hat{\theta} .
$$

Table 1

CALCULATION OF ODDS RATIOS

Sample 1 Sample 2

$\begin{array}{lll}\text { Response }=1 & n_{11} & n_{21} \\ \text { Response }=2 & n_{12} & n_{22} \\ \text { Totals } & n_{1} & n_{2}\end{array}$

Taking the $\log$ of $\hat{\theta}$ makes the choice of which group is Sample 1 and which is Sample 2 irrelevant, at least in absolute value.

Let us calculate and examine the odds ratio of two hypothetical samples. Consider the situation where both $n_{1}$ and $n_{2}$ are 10,000. Let Sample 1 and Sample 2 claim 1,000 and 2,000 1-responders, respectively. The odds of a 1 -responder from Sample 1 is thus $\frac{1}{9}$ and likewise, the odds of a 1-responder from Sample 2 is $\frac{2}{8}$. Further, the odds ratio is $\frac{4}{9}$ and the $\log$ of the odds ratio is $\ln \frac{4}{9}=-0.811$. If we were to switch the labels of the samples, the odds ratio would have been $\frac{9}{4}$ with $\log 0.811$. Thus, the labelling of the samples affects only the sign of the log of the odds ratio.

The Appendix includes the derivation of the asymptotic distribution of the log of the odds ratio. The large-sample distribution of $\hat{\lambda}$ is shown to be normal with mean $\lambda$ and variance, $\sigma^{2}$. A consistent estimator of $\sigma^{2}$ is found to be:

$$
\hat{\sigma}^{2}=\frac{1}{n_{11}}+\frac{1}{n_{21}}+\frac{1}{n_{12}}+\frac{1}{n_{22}} .
$$


Gart notes that the estimator $\hat{\theta}$ is biased due to the fact that its expectation is infinite because one of the terms in the denominator is zero with positive probability. ${ }^{4}$ This probability vanishes for large sample sizes. The Haldane-Ascombe correction of $\frac{1}{2}$ yields the following approximately unbiased estimator: ${ }^{5}$

$$
\hat{\lambda}_{\mathrm{HA}}=\ln \hat{\theta}_{\mathrm{HA}}=\ln \frac{\left(n_{11}+\frac{1}{2}\right)\left(n_{22}+\frac{1}{2}\right)}{\left(n_{21}+\frac{1}{2}\right)\left(n_{12}+\frac{1}{2}\right)} .
$$

Further, Gart and Zweifel ${ }^{6}$ recommend incorporating the correction of $\frac{1}{2}$ into the expression for the asymptotic variance of $\hat{\lambda}_{\mathrm{HA}}$,

$$
\begin{aligned}
\hat{\sigma}_{\mathrm{HA}}^{2}= & \frac{1}{n_{11}+\frac{1}{2}}+\frac{1}{n_{12}+\frac{1}{2}}+\frac{1}{n_{21}+\frac{1}{2}} \\
& +\frac{1}{n_{22}+\frac{1}{2}},
\end{aligned}
$$

which still yields a consistent estimator, but avoids the possibility of dividing by 0 .

Further on this latter version will be used. The subscript HA will be omitted for the remainder of the paper.

\section{The z-statistic for comparisons}

of odds ratios in relation to offer preference modelling

Thus far the discussion of odds ratios has been limited to single calculations of it. The purpose, however, is to segment a population into groups such that the difference in odds ratios is maximised. The discussion must therefore be extended to comparisons of odds ratios over subsets of the population. This section motivates and introduces a split statistic, which is utilised to segment the population.

Consider a split on the data which divides a sample space $T$ into two groups $S$ and $S^{\prime}$ such that $S$ and $S^{\prime}$ are mutually exclusive and collectively exhaustive subsets of $T$. Examples of possible splits in a direct marketing environment are:

- women vs. men and customers of unknown gender

- customers with two or more purchases vs. those with zero or one

- new customers vs. longstanding customers.

Further, generate $2 \times 2$ contingency tables based on two indicator variables within both $S$ and $S^{\prime}$. The data can be displayed as in Table 2. One indicator is the response indicator, and the other is the offer indicator. Thus the value $n_{11}$ represents the number of Offer 1 responders in $S$, the value of $n_{21}^{\prime}$ represents the number of Offer 2 responders in $S^{\prime}$, etc.

Let

$$
\begin{aligned}
\hat{\lambda}_{S}= & \ln \frac{\left(n_{11}+\frac{1}{2}\right)\left(n_{22}+\frac{1}{2}\right)}{\left(n_{21}+\frac{1}{2}\right)\left(n_{12}+\frac{1}{2}\right)} \\
\hat{\sigma}_{S}^{2}= & \frac{1}{n_{11}+\frac{1}{2}}+\frac{1}{n_{12}+\frac{1}{2}}+\frac{1}{n_{21}+\frac{1}{2}} \\
& +\frac{1}{n_{22}+\frac{1}{2}} \\
z= & \frac{\hat{\lambda}_{S}-\hat{\lambda}_{S^{\prime}}}{\sqrt{\hat{\sigma}_{S}^{2}+\hat{\sigma}_{S^{\prime}}^{2}}}
\end{aligned}
$$


Table 2

CALCULATION OF TEST STATISTIC FOR DIFFERENCES IN ODDS RATIOS

\begin{tabular}{lllll}
\hline & $\begin{array}{l}\text { Members of } S \\
\text { Offer 1 }\end{array}$ & Offer 2 & Members of $S^{\prime}$ & \\
& & & Offer 1 & Offer 2 \\
Response & $n_{11}$ & $n_{21}$ & $n^{\prime}{ }_{11}$ & $n^{\prime}{ }_{21}$ \\
Non-response & $n_{12}$ & $n_{22}$ & $n^{\prime}{ }_{12}$ & $n^{\prime}{ }_{22}$ \\
\hline
\end{tabular}

Equation (3) above is identical to Equation (1) previously, the assumed difference being that it is calculated on a fraction of the sample, denoted by the subscript $S$. Evidently, under $H_{0}$ : $\lambda_{S}=\lambda_{S^{\prime}}, \quad z \rightarrow N(0,1)$. Thus, to test if the $\operatorname{logs}$ of the odds ratios are significantly different within the two subsamples, the $z$-statistic is checked against the values in the standard normal distribution tables. Extreme values of $z$ indicate a significant difference.

Let us consider the value of $\hat{\lambda}_{S}$ for our problem. The value of 0 is only possible if $n_{11} / n_{12}=n_{21} / n_{22}$, or the ratio of responders to non-responders is the same for both Offer 1 and Offer 2 categories within subsample $S$. Higher values of $\hat{\lambda}_{S}$ are found as $n_{11} / n_{12}$ is greater than $n_{21} / n_{22}$. This implies that Offer 1 recipients are more likely to respond than Offer 2 recipients within subsample $S$. Negative values of $\hat{\lambda}_{S}$ would imply that the response rate within the Offer 2 group is higher than in the Offer 1 group. The calculation of $\hat{\lambda}_{S^{\prime}}$ is the same as for $\hat{\lambda}_{S}$, and the above statements also hold for $\lambda_{S^{\prime}}$.
The $z$-statistic will take on values near to 0 when the values of $\hat{\lambda}_{S}$ are near to the values of $\hat{\lambda}_{S^{\prime}}$. This implies that the $S / S^{\prime}$ split does not discriminate well between people more likely to respond to one promotion or the other. When $z$ has significantly positive values, those in $S$ display more of a preference for Offer 1 than those in $S^{\prime}$. The opposite is true for significantly negative values of $z$, in that case, members of $S^{\prime}$ have more preference for Offer 2 than members of $S$.

\section{A TECHNIQUE FOR IDENTIFYING OFFER PREFERENCE MODELLING VARIABLES AND CREATING OPTIMAL DUMMIES: TaCT}

The technique discussed in this section is a variation on the CART algorithm developed by Breiman et al. ${ }^{7}$ CART is a binary tree structured classification technique. Thorough examinations of CART and its application to response modelling in direct marketing 
Table 3

\begin{tabular}{|c|c|c|c|c|c|c|}
\hline & TABULAT & $\mathrm{ON}$ OF A & CANDI & ITE SPLIT & VARIAB & \\
\hline Sex & $\begin{array}{l}\text { Offer } 1 \\
\text { Responder }\end{array}$ & $\begin{array}{l}\text { Offer } 1 \\
\text { Non- } \\
\text { Responder }\end{array}$ & $\begin{array}{l}\text { Offer } 1 \\
\text { Response } \\
\text { Rate }\end{array}$ & $\begin{array}{l}\text { Offer } 2 \\
\text { Responder }\end{array}$ & $\begin{array}{l}\text { Offer } 2 \\
\text { Non- } \\
\text { responder }\end{array}$ & $\begin{array}{l}\text { Offer } 2 \\
\text { Response } \\
\text { Rate }\end{array}$ \\
\hline Women & 88 & 1,043 & 7.78 & 92 & 1,174 & 7.27 \\
\hline Men & 294 & 4,592 & 6.02 & 153 & 4,402 & 3.36 \\
\hline Unknown & 77 & 765 & 9.14 & 61 & 895 & 6.38 \\
\hline Total & 459 & 6,400 & 6.69 & 306 & 6,471 & 4.52 \\
\hline
\end{tabular}

are found in Haughton and Oulabi ${ }^{8}$ and Thrasher. ${ }^{9}$ The differences between this new method and CART lies in the purpose of classification and thereby also in the mode of splitting nodes. CART is a classification tool. The new tree segmentation technique divides analysis samples according to their responses to two treatments, such that the resultant groups display differing response rates to those treatments.

Briefly, tree-structured classifiers are developed by performing repeated binary partitions on the data in an effort to identify segments with a particular behaviour or classification. Breiman et al. state that there are three central elements to building tree-structured classifiers: (1) the determination of the splits; (2) the decision as to when splitting or partitioning should cease; and (3) the classification of each final subgroup into a class. ${ }^{10}$ These three elements will be referred back to as the new technique is outlined.

The first element refers to the selection of splits. Using the $z$-statistic discussed in the previous section as a split statistic, the algorithm searches through all possible binary splits on the data of the following types:

- for categorical variables, all combinations of values

- for continuous variables, all splits of the form, $x_{i} \in(-\infty, c]$ or $x_{i} \in(c, \infty)$ for all $c$,

for the split yielding the $z$-statistic with the maximum absolute value. In other words, the technique searches for the split on the data which produces subgroups with the most significant difference between their odds ratios. Seeking out splits on continuous variables may appear to be a daunting (if not impossible) task, however, the 
Figure 1: Offer 1 and Offer 2 response rates by split variable

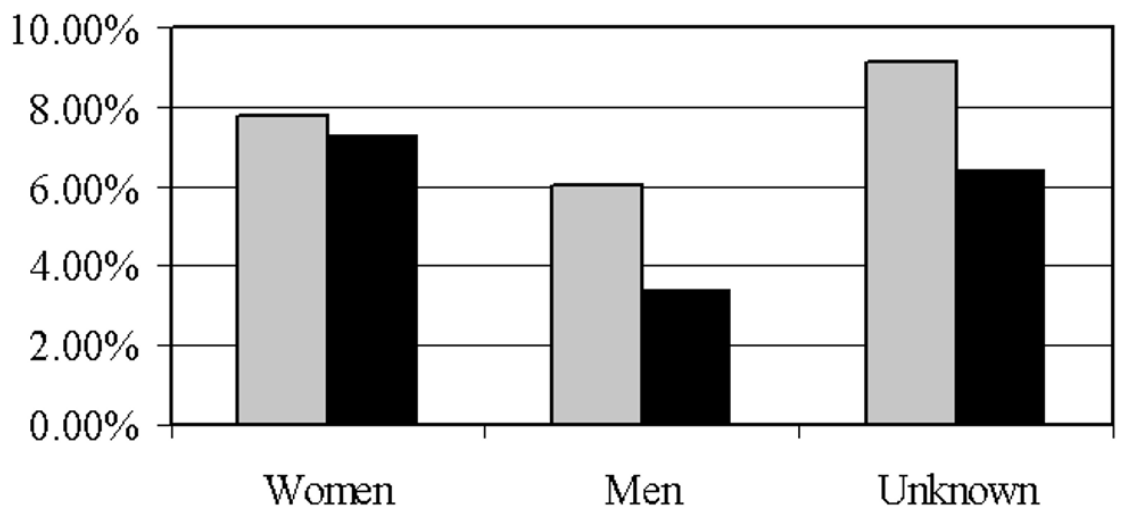

Response Rate Offer 1 Response Rate Offer 2

search algorithm can be coded so that the real line is reduced to only those values of the continuous variable that exist on the data set at hand.

The idea will be explained with an example. The split variable examined is categorical, meaning that all combinations of categories will be considered. The split variable represents the sex of the customer. Table 3 displays the data. The number of responders, non-responders, and the order rates are given for each value of the split variable.

Figure 1 shows a graph of the response rates. Note that women have a similar response rate given either Offer 1 or Offer 2. Men and customers for which sex is unknown, however, react much more strongly to Offer 1 than to Offer 2. In addition, women appear more likely to order from either offer than men. The focus, however, is not on the relation between response rate and sex, but on the relation between the response rates to the two offers for each sex. Stated simply: we seek to understand the interaction between offer and the sex of the customer.

Note how difficult it would be to find by eye a binary split on the data such that the separation of the odds ratios would be maximised. By defining a split criterion, the $z$-statistic, and applying it to all possible splits, tedious examinations of tabulations can be avoided.

There are three possible splits on this variable: women vs. others, men vs. others, and men and women vs. those of unknown sex. The algorithm 
Figure 2: Resulting tree - Splitting on sex

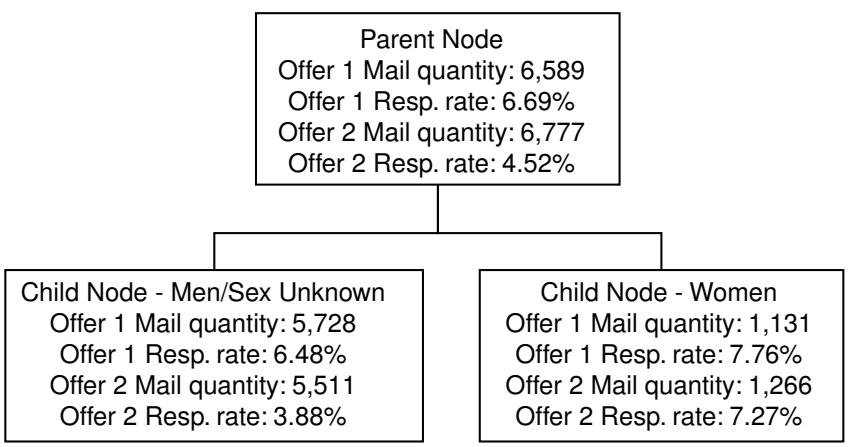

calculates the split statistic for each of the three splits. Each of the $z$-statistics is shown in Table 4. The best split is between women and others, where the $z$-statistic takes on a value of -2.60 . The tree would appear as shown in Figure 2.

The parent node is the original group, and the child nodes are created by applying the most significant split of the parent node. In this case the best

\section{Table 4}

\section{z-STATISTICS FOR SPLITS ON SEX}

Split $\quad z$-statistic

$\begin{array}{lr}\text { Women vs. others } & -2.60 \\ \text { Men vs. others } & 2.57 \\ \text { Unknown vs. others } & -0.23\end{array}$

split yields one child node with very similar response rates to the two offers. The other child node shows an even greater relative preference for Offer 1 over Offer 2 than the parent node exhibits.

If we were to repeat the process for each child node, possibly splitting on other variables, large trees result. Hence, at each node the split with the highest $z$-statistic is sought out, the node is partitioned according to that split and each child node is treated subsequently as a parent node.

The paper now concentrates on the second of Breiman et al's elements, namely, the decision when to cease the node splitting process. The author suggests looking at the $p$-values of the split with the highest absolute value for $z$ for any given node. Once the maximum $p$-value slips into the nonsignificant range, which can be determined by the modeller, splitting would stop for that node. She also suggests 
that splitting stop when the nodes contain too few observations.

Finally, there is the third of Breiman et al's elements: the decision on how to classify terminal nodes into groups. More specifically, how to divide consumers into offer groups for direct mail. Two strategies arise, classify nodes such that:

- response is maximised, or - profit is maximised.

As a first step toward deriving the offer assignment rules, define the following quantities:

$$
\begin{aligned}
C_{1}= & \text { the cost of Offer } 1 \\
C_{2}= & \text { the cost of Offer } 2 \\
m_{1}= & \text { the margin of Offer } 1 \\
m_{2}= & \text { the margin of Offer } 2 \\
\hat{p}_{1}= & \text { the observed response rate } \\
& \text { within the node for Offer } 1 \\
\hat{p}_{2}= & \text { the observed response rate } \\
& \text { within the node for Offer } 2 .
\end{aligned}
$$

If response is to be maximised, the node should be classified to the offer for which the observed response rate is higher. Hence, assign the node to Offer 1 if $\hat{p}_{1}>\hat{p}_{2}$, otherwise assign it to Offer 2. Maximising response rather than profit is often the goal of direct campaigns selling magazine subscriptions, where a certain quantity of active subscribers is guaranteed to advertisers.

When profits drive the assignment, the rule is more complex. The estimated profit per offer recipient, $\hat{R}_{1}$ and $\hat{R}_{2}$, for Offers 1 and 2 , respectively, can be calculated as follows:

$$
\begin{aligned}
& \hat{R}_{1}=\hat{p}_{1} m_{1}-C_{1} \\
& \hat{R}_{2}=\hat{p}_{2} m_{1}-C_{2}
\end{aligned}
$$

The rule is then simply to classify the node to Offer 1 if $\hat{R}_{1}>\hat{R}_{2}$, otherwise assign it to Offer 2 . If the offer costs and margins are the same for both offers, the two rules are equivalent.

Breiman et al. also address extensively the issue of estimating the error rates for classification trees obtained using CART. They advocate two methods of error estimation, namely by using a hold-out sample, and by iteratively holding out one observation, fitting a model, and then tallying whether the holdout observation is correctly or incorrectly classified. The tree-based technique described in this paper does not lend itself to the latter of Breiman et al's methods. The reason is that the problem is not a pure classification problem. Groups of customers can only be classified as identified by relevant characteristics into an offer segment based on a comparison of order rates between samples receiving varying offers. Thus, the correctness of the assignment cannot be determined given a single observation. The author therefore recommends using a holdout sample for validation.

\section{ILLUSTRATIVE EXAMPLE}

When does TaCT outperform logit regression with interaction variables? If one includes all possible interactions 
Figure 3: Illustrative case tree diagram

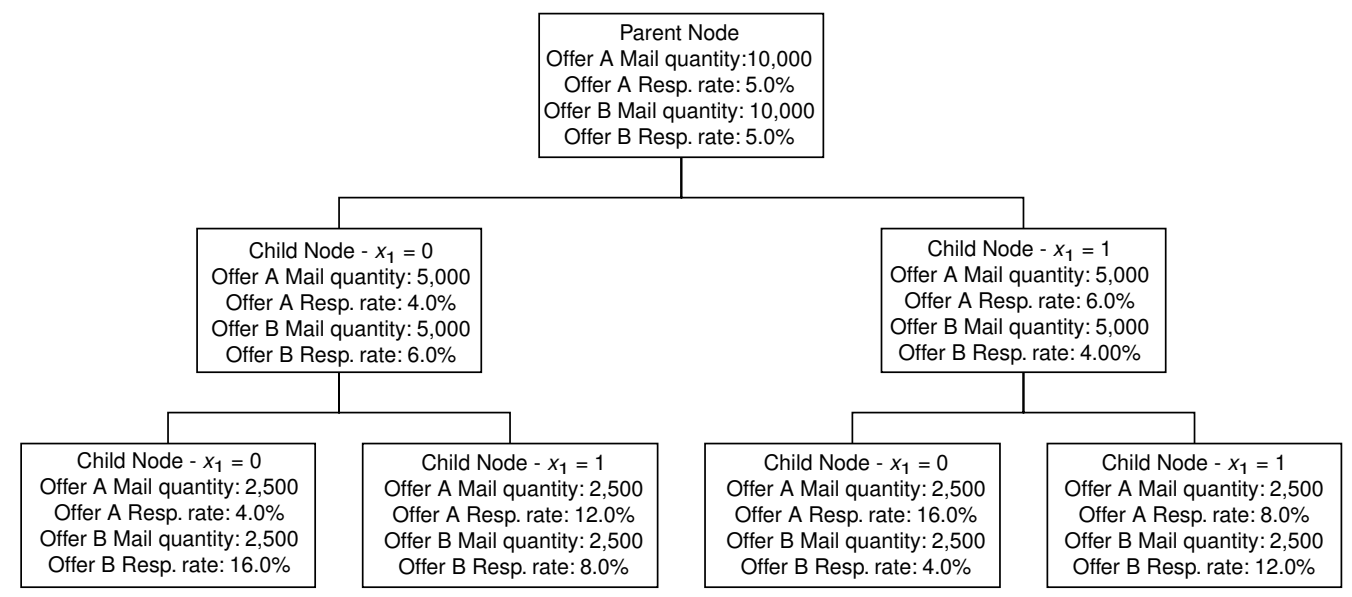

in the logit model, TaCT will not produce a more profitable segmentation. Including all interactions is a daunting task, however, especially considering the volume of data available on DM databases. TaCT reduces the task of modelling by finding which independent variables are interacting with offer type in predicting response, and also finding higher order interactions involving two or more independent variables and offer type.

Breiman et al. cite the conditions under which their own CART model is preferred to regression. One of these conditions is non-homogeneity in the data. That is, where different relations hold over different partitions of the sample space. Thus, the originators of CART also were concerned with interactions when they suggested their tree-based algorithm.
Consider the following example. The data here are constructed to be illustrative. Tests of Offer A and Offer $\mathrm{B}$ are each sent to 10,000 people. The tests each yield a response rate of five per cent. There are two binary independent characteristics present, $x_{1}$ and $x_{2}$. TaCT produces the model shown in Figure 3.

The first split is performed on variable $x_{1}$, which yields two segments. The left node of the first split prefers Offer B and the right node prefers Offer A. The second split is performed on variable $x_{2}$. Here interaction is present. Depending on the values of $x_{1}$, the values of $x_{2}$ exert different effects on the preference for Offer A over Offer B. Customers with a value of one for $x_{2}$ prefer Offer $\mathrm{A}$ if their value for $x_{1}$ is zero, but they prefer Offer $\mathrm{B}$ if their value for $x_{1}$ is one. 
Table 5

\section{COMPARISON OF LOGIT REGRESSION COEFFICIENTS}

Coefficients

Model 1 Model 2 Model 3

\begin{tabular}{lrrr} 
Offer & 0.000 & 0.450 & 1.520 \\
$x_{1}$ & 0.000 & 0.450 & 1.520 \\
$x_{2}$ & 0.000 & 0.000 & 1.186 \\
Offer $x_{1}$ & $\mathrm{n} / \mathrm{a}$ & -0.900 & -3.040 \\
Offer $x_{2}$ & $\mathrm{n} / \mathrm{a}$ & 0.000 & -1.970 \\
$x_{1} x_{2}$ & $\mathrm{n} / \mathrm{a}$ & $\mathrm{n} / \mathrm{a}$ & -1.970 \\
Offer $x_{1} x_{x_{2}}$ & $\mathrm{n} / \mathrm{a}$ & $\mathrm{n} / \mathrm{a}$ & 3.940 \\
Intercept & -2.197 & -2.442 & -3.178 \\
\hline
\end{tabular}

Using TaCT, one can segment the customer population according to offer preference. The rule of response maximisation is applied. Nodes 2 and 3 would thus be assigned to the Offer A segment and Nodes 1 and 4 would be segmented into the Offer B group.

Let us apply a logit model to the data illustrated above. Three models will be fitted. The first includes just an offer indicator (equals 1 for Offer A, otherwise 0 ), $x_{1}$ and $x_{2}$ as independent variables. The second models response as a function of offer, $x_{1}, x_{2}$, and the interaction of $x_{1}$ with offer and the interaction of $x_{2}$ with offer. The third model includes the offer indicator, $x_{1}$, $x_{2}$, and all possible interactions of these three variables. Table 5 displays the coefficients for the three models.

Model 1 shows only one significant coefficient, that of $x_{1}$. The first order terms Offer and $x_{2}$ do not discriminate responses. The coefficients of Offer, $x_{1}$, and Offer $x_{1}$ are significant in Model 2 , but $x_{2}$, and the interaction between Offer and $x_{2}$ are not significant predictors of response rate for this sample. Model 3 shows all variables adding a significant discriminating power.

Were these models to be used to predict response rate for the two offers given values of $x_{1}$ and $x_{2}$, the results would be as given in Table 6 .

Model 1 shows no discrimination between response to Offer A or Offer B. Model 2 is somewhat more successful. If we utilise its results, we arrive at the same segmentation produced by the first split in the tree of Figure 3. Model 3 exactly reproduces the segmentation of the tree diagram, but with notably more work.

\section{CASE STUDY}

In this section the technique described above is used on two offer tests. Two random samples of approximately 14,000 names were taken simultaneously from a single customer population. Offer 2 did not generate as many responses (orders) as Offer 1 . The exact mail quantities, response frequencies and response rates are shown in Table 7 . The combined samples were split into two approximately equal-sized groups, one for analysis and the other for validation.

The tree-generating procedure was run on the dataset with 11 predictive variables. The resulting tree is shown 
Table 6

PREDICTED RESPONSE RATES FOR THREE LOGIT MODELS

\begin{tabular}{|c|c|c|c|c|c|c|}
\hline \multirow[b]{3}{*}{$x_{1}$} & \multirow{2}{*}{\multicolumn{2}{|c|}{$\begin{array}{l}\text { Model } 1 \\
\text { Predicted response } \\
\text { rate given Offer }\end{array}$}} & \multirow{2}{*}{\multicolumn{2}{|c|}{$\begin{array}{l}\text { Model } 2 \\
\text { Predicted response } \\
\text { rate given Offer }\end{array}$}} & \multirow{2}{*}{\multicolumn{2}{|c|}{$\begin{array}{l}\text { Model } 3 \\
\text { Predicted response } \\
\text { rate given Offer }\end{array}$}} \\
\hline & & & & & & \\
\hline & A & $\mathrm{B}$ & A & $\mathrm{B}$ & A & B \\
\hline 0 & 0.1 & 0.1 & 0.12 & 0.08 & 0.16 & 0.04 \\
\hline 0 & 0.1 & 0.1 & 0.08 & 0.12 & 0.04 & 0.16 \\
\hline 1 & 0.1 & 0.1 & 0.12 & 0.08 & 0.08 & 0.12 \\
\hline 1 & 0.1 & 0.1 & 0.08 & 0.12 & 0.12 & 0.08 \\
\hline
\end{tabular}

in Figure 4. The terminal nodes are marked by a letter in the node. The company which supplied the data requested that details about the offers and the further variables on which the splits were performed not be disclosed.

The first split is the one which was used to illustrate the technique (see Figure 2). The second split, with

\section{Table 7}

\section{SAMPLES INFORMATION}

\begin{tabular}{llll}
\hline & $\begin{array}{l}\text { Mail } \\
\text { quantity } \\
\text { (MQ) }\end{array}$ & Responders & $\begin{array}{l}\text { Response } \\
\text { rate }\end{array}$ \\
Offer & & & \\
Offer 1 & 13,969 & 901 & 6.45 \\
Offer 2 & 13,708 & 611 & 4.46 \\
\hline
\end{tabular}

parent node having 5,728 Offer $1 \mathrm{MQ}$ (mail quantity), yields two partitions, one of which (terminal node C) shows a preference for Offer 2. The Offer 2 response rate is almost double that of Offer 1, even though the customers in the parent node on the whole prefer Offer 1.

Offer 1 costs NLG 2.29 and Offer 2 costs slightly less, at NLG 1.64. The margin expected from a responder to Offer 1 and 2 was NLG 67.61 and NLG 72.66, respectively. The cost and margin figures can be combined with the results to determine the optimal classification for each terminal node according to profits. Thus the nodes A, $\mathrm{C}$ and $\mathrm{D}$ to Offer 1 and nodes $\mathrm{B}, \mathrm{E}$, and $F$ G to Offer 2 were classified. If one wished to maximise the response rates, then nodes $\mathrm{A}, \mathrm{C}, \mathrm{D}$ and $\mathrm{F}$ would be given Offer 1, with the others receiving Offer 2. Given no selectivity, 
Figure 4: Tree diagram on analysis sample

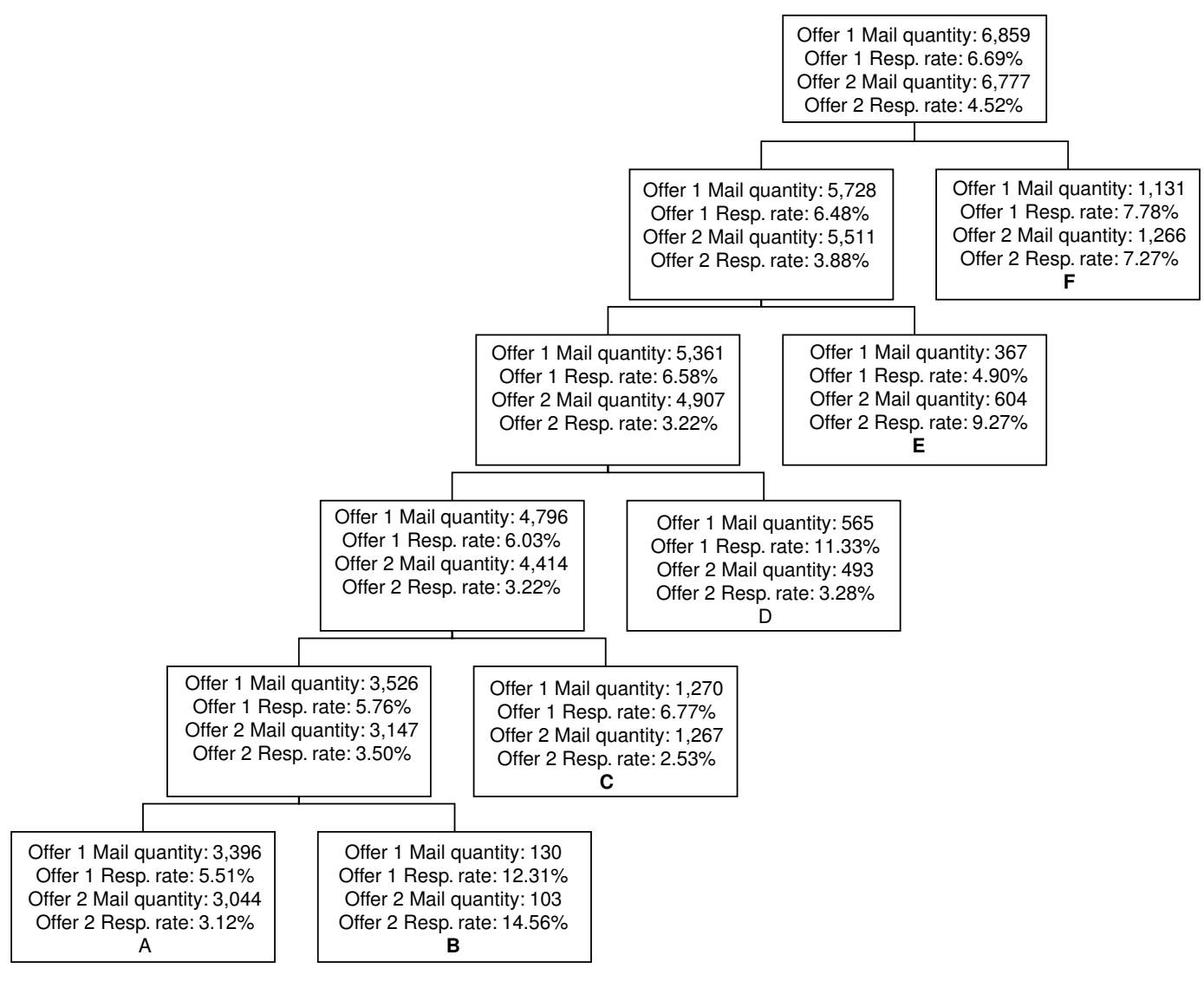

Offer 1 would be chosen for both the response maximising strategy and the profit maximising strategy. Table 8 displays the mail quantities, order rates and profit projections by offer and node. The figures in Table 8 were taken from the validation set, the tree and classification of nodes having been performed on the analysis set.

Selectivity based on response yields a validation order rate of 6.50 per cent, very slightly above that of Offer 1 on the whole of 6.22 per cent. Selectivity based on profit yields a profit of NLG $17,699.33$, which is 30 per cent higher than the profit yielded by an Offer 1 only strategy. Thus, it has been shown that $\mathrm{TaCT}$ can be a useful tool for a direct marketing statistician, and can increase profits significantly.

A logit model fit on the same analysis sample yields a profit of NLG 


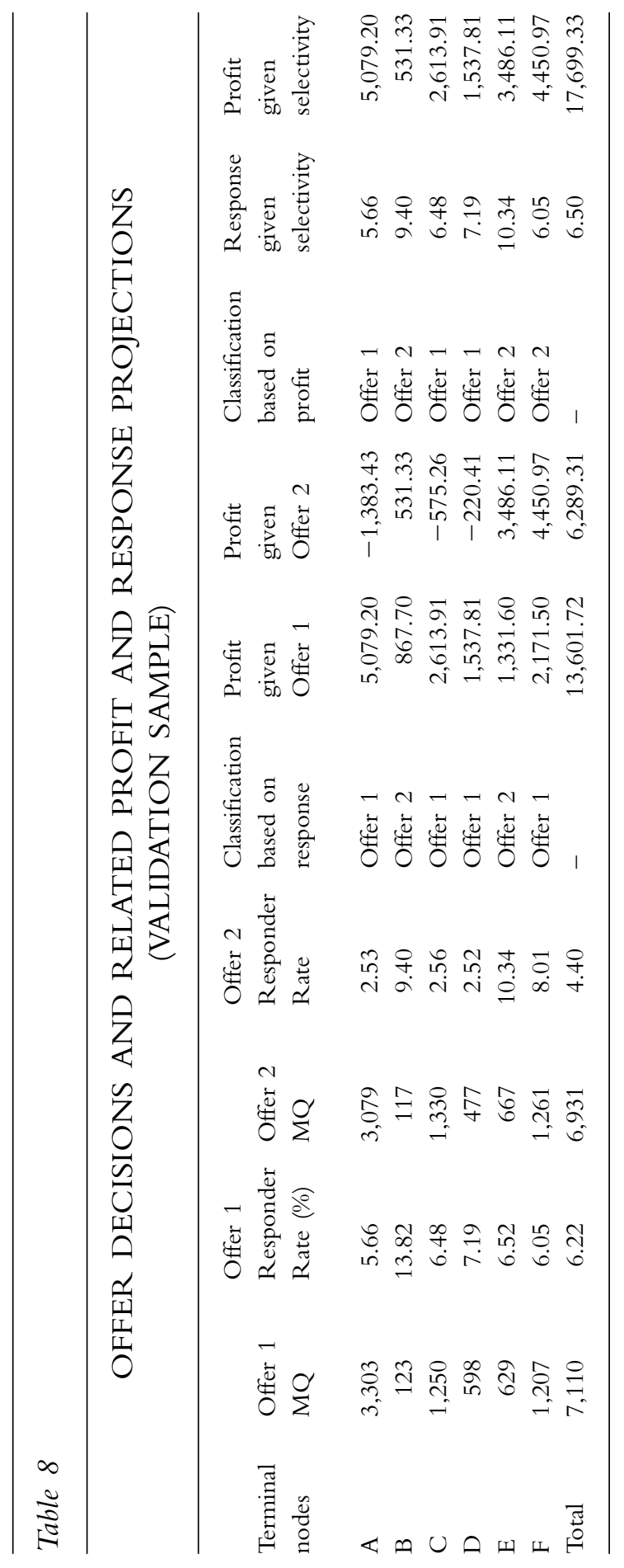


$18,188.53$ on the validation sample, an increase of 2.7 per cent over the profit attained using the tree's solution. The variables included in that model were the same as those used in the treebased analysis, the offer indicator, and all two-way interactions between the independent variables and the offer indicator. Three of the coefficients are non-significant. If response maximisation is used to determine offer segmentation the validation response rate yielded is 6.93 per cent, a very slight increase over TaCT's result. It appears that there is not enough nonhomogeneity in the data for the tree model to yield a superior solution. Only variables deemed significant by the tree model into the logit model were incorporated, however.

If one employs a stepwise procedure to select variables for the logit model, the results are quite different. The validation profit of such a model is NLG $13,646.17$, or only about 77 per cent of the profit obtained utilising the tree method. It appears that TaCT's results at least approach those of a logit model with interaction variables, and that the tree model is useful for determining which interactions are important.

In the course of developing and testing $\mathrm{TaCT}$, the team encountered situations where the tree segmentation performed worse than no segmentation on validation samples. These are described below along with suggestions on how to best use the technique.

It is very important that the bulk response rates mirror those of the tests being analysed. The node classification process relies heavily on the relation between the response rates of the two tests. If the marketing situation changes, and one offer is far less (or more) effective than shown in the test, then the classifications will certainly be suboptimal. As with any tree-based model, one should attempt to understand the meaning behind the splits found. In this way, it is possible to foresee if the split is trustworthy, and if the relation can be expected to repeat itself in the bulk mailing. Do this especially for small nodes with large differences in response rates between the offers. It is wise to avoid producing very small nodes.

\section{BUSINESS IMPLICATIONS AND FUTURE STEPS}

This paper has introduced a method for identifying customer segments that show a preference for one type of offer over another. The differences in the two offers might involve any element, such as product, channel (direct mail vs. telemarketing), premium, price, brochure etc. By more closely matching offers to consumers' preferences, direct marketers can increase their profits while also increasing the satisfaction of their customers.

An increase in the number of available offers for a given campaign will ultimately yield greater variety for the customers. If one type of appeal is unsuccessful with certain consumers, a different offer can be made in the next campaign. Focusing creative 
efforts just on one offer at a time can force companies into a narrow strategy, which considers only the offer preference of the majority. By utilising a narrow offer strategy, companies can lose sales to consumers not moved by that strategy, and can lose customers as they become bored with repeatedly receiving the same type of offers. By approaching consumers with the offer they will consider most appealing, a greater number of sales can be made.

The purpose of $\mathrm{TaCT}$ is to utilise offer tests to segment a customer base according to offer preference and ultimately increase profits. Many direct marketing companies perform offer tests for the purpose of choosing one for a bulk campaign. Given the existence of these tests, the use of the technique outlined in this paper does not require investment in advance. Increased costs to the direct marketer may be incurred however, in that two offers must be produced for a campaign, rather than one.

The author believes there are two directions in which this research leads. One involves the extension of the technique itself, and the other involves its application.

The presentation of the technique in this paper displayed only two tests in each instance. Indeed, by their nature, odds ratios can only be calculated for two tests at a time. One can, however, imagine the situation that a marketer would like to segment a list into three or more offer groups. Berger and Magliozzi suggested factorial offer testing design, with four or more tests being performed with at least two levels of two elements. ${ }^{11}$ An analysis technique which could use such test data for segmentation would be potentially very profitable when there is interaction also between the elements in the offers being tested.

On the application side, much research needs to be done to determine which characteristics are trustworthy for offer segmentation. Direct marketers know the value of recency-frequency-monetary variables in response modelling. For offer segmentation, however, the relationships between offer preference and past performance are not so clear. For instance, imagine a marketer trying to decide which customers are to receive an offer which promises a premium with the order and which customers are to receive the same offer without the premium. S/he could reason that a customer with a very high frequency of products paid is interested in the products, not the premiums, and thus should receive the offer with no premium. On the other hand, if the direct marketing firm often sends offers with premiums promised, the premiums might be the very reason the customer orders so frequently and therefore frequent customers should receive offers with premiums. It seems that both conclusions regarding premium offers to frequent customers can be rationalised. A thorough validation of candidate partition variables is advocated before an offer segmentation analysis be adopted in a bulk mailing. 


\section{ACKNOWLEDGEMENT}

The author would like to thank Tom Wansbeek, Ton Steerneman and two anonymous reviewers for their helpful comments.

\section{REFERENCES}

1 Bult, J. R., van der Scheer, H. R. and Wansbeek, T. J. (1997) 'Interaction between target and mailing characteristics in direct marketing, with an application to health care fund raising', International Journal of Research in Marketing, Vol. 14, pp. 301-308.

2 Spring, P. N., Leeflang, P. S. H. and Wansbeek, T. J. (1999) 'The combination strategy to simultaneous target selection and offer segmentation', Journal of Market Focused Management, Vol. 4, pp. 187-203.

3 Breiman, L., Friedman, J., Olshen, R. and Stone, C. (1984) 'Classification and regression trees', Wadsworth, Monterey, CA.

4 Gart, J. J. (1971) 'The comparison of proportions: A review of significance tests, confidence intervals and adjustments for stratification', Review of the International Statistical Institute, Vol. 39, pp. 148-169.

5 Haldane, J. B. S. (1955) 'The estimation and significance of the logarithm of a ratio of frequencies', Annals of Human Genetics, Vol. 20, pp. 309-311.

6 Gart, J. J. and Zweifel, J. R. (1967) 'On the bias of various estimators of the logit and its variance with application to quantal bioassay', Biometrika, Vol. 54, pp. 181-187.

7 Breiman et al. (1984) op. cit.
8 Haughton, D. and Oulabi, S. (1993) 'Direct marketing modeling with CART and CHAID', Journal of Direct Marketing, Vol. 7, No. 3, pp. 16-26.

9 Thrasher, R. P. (1993) 'CART: A recent advance in tree-structured list segmentation methodology', Journal of Direct Marketing, Vol. 5, pp. 35-47.

10 Breiman et al. (1984) op. cit.

11 Berger, P. D. and Magliozzi, T. L. (1993) 'Experimental design in direct mail and the application of Taguchi methods', Journal of Direct Marketing, Vol. 7, pp. 44-54.

\section{APPENDIX}

In this appendix the asymptotic distribution of the $\log$ of the odds ratio, $\hat{\lambda}$, is derived. The asymptotic distribution of $\hat{\lambda}$ is needed in order to derive the test for the significance of differences in $\hat{\lambda}$ within segments of the populations. The statistic $\hat{\lambda}$ is a function of $\pi_{11}$ and $\pi_{21}$, both of which are asymptotically normal.

The delta method is a procedure for deriving the asymptotic distribution of an estimator which is a function of an asymptotically normally distributed statistic. Assume that a statistic $T_{m}$ is approximately normally distributed with mean $\theta$ and various $\sigma^{2} / m$, where $m$ denotes sample size, or alternatively $\sqrt{m}\left(T_{m}-\theta\right) \rightarrow N\left(0, \sigma^{2}\right)$. Let $g$ be a function of $T_{m}$. Given that $g$ is twice differentiable at $\theta$, we have the following result:

$$
\sqrt{m}\left[g\left(T_{m}\right)-g(\theta)\right] \stackrel{d}{\rightarrow} N\left(0, \sigma^{2}\left[g^{\prime}(\theta)\right]^{2}\right) .
$$


The result (1) is known as the delta method for deriving asymptotic distributions.

We are interested in the asymptotic distribution of $\hat{\lambda}$. Since

$$
\begin{aligned}
\lambda= & \ln \frac{\hat{\pi}_{11} \hat{\pi}_{22}}{\hat{\pi}_{12} \hat{\pi}_{21}} \\
= & \ln \hat{\pi}_{11}-\ln \hat{\pi}_{12}+\ln \hat{\pi}_{22}-\ln \hat{\pi}_{21} \\
= & \ln \hat{\pi}_{11}-\ln \left(1-\hat{\pi}_{11}\right)+\ln \hat{\pi}_{22} \\
& -\ln \left(1-\hat{\pi}_{22}\right)
\end{aligned}
$$

and since $\pi_{11}$ and $\pi_{22}$ are independent,

$$
\begin{aligned}
\operatorname{var}(\hat{\lambda})= & \operatorname{var}\left(\ln \hat{\pi}_{11}-\ln \left(1-\hat{\pi}_{11}\right)\right) \\
& +\operatorname{var}\left(\ln \hat{\pi}_{22}-\ln \left(1-\hat{\pi}_{22}\right)\right)
\end{aligned}
$$

and the derivations for the two terms on the right hand side of equation (2) are identical. Since

$$
\sqrt{n_{1}}\left(\hat{\pi}-\pi_{11}\right) \stackrel{d}{\rightarrow} N\left(\pi_{11}, \pi_{11} \pi_{12}\right)
$$

implies, by means of invoking the delta method,

$$
\begin{aligned}
a_{1} \equiv & \sqrt{n_{1}}\left[\ln \hat{\pi}_{11}-\ln \left(1-\hat{\pi}_{11}\right)\right. \\
& \left.-\ln \pi_{11}-\ln \left(1-\pi_{11}\right)\right] \\
a_{1} \stackrel{d}{\rightarrow} & N\left(0, \pi_{11} \pi_{12}\right. \\
& \left.\times\left(\frac{\partial\left(\ln \pi_{11}-\ln \left(1-\pi_{11}\right)\right)}{\partial \pi_{11}}\right)^{2}\right) \\
= & N\left(0, \frac{1}{\pi_{11}}+\frac{1}{\pi_{21}}\right) .
\end{aligned}
$$

Likewise,

$$
\begin{aligned}
a_{2} \equiv & \sqrt{n_{2}}\left[\ln \hat{\pi}_{22}-\ln \left(1-\hat{\pi}_{22}\right)\right. \\
& \left.-\ln \pi_{22}-\ln \left(1-\pi_{22}\right)\right] \\
a_{2} \stackrel{d}{\rightarrow} & N\left(0, \pi_{21} \pi_{22}\right. \\
& \left.\times\left(\frac{\partial\left(\ln \pi_{22}-\ln \left(1-\pi_{22}\right)\right)}{\partial \pi_{22}}\right)^{2}\right) \\
= & N\left(0, \frac{1}{\pi_{21}}+\frac{1}{\pi_{22}}\right) .
\end{aligned}
$$

Let us assume that $n_{1} / n_{2} \rightarrow k^{2}$, such that $0<k^{2}<\infty$. We can thus write:

$$
\begin{aligned}
\sqrt{n_{1}}(\hat{\lambda}-\lambda)= & a_{1}+\frac{\sqrt{n_{1}}}{\sqrt{n_{2}}} a_{2} \\
\stackrel{d}{\rightarrow} & N\left(0, \frac{1}{\pi_{11}}+\frac{1}{\pi_{21}}+k^{2}\right. \\
& \left.\times\left(\frac{1}{\pi_{21}}+\frac{1}{\pi_{21}}\right)\right)
\end{aligned}
$$

so

$$
\begin{aligned}
\hat{\lambda} \stackrel{\text { appr. }}{\sim} & N\left(\lambda, \frac{1}{n_{1}}\left(\frac{1}{\pi_{11}}+\frac{1}{\pi_{21}}+\frac{n_{1}}{n_{2}}\right.\right. \\
& \left.\left.\times\left(\frac{1}{\pi_{21}}+\frac{1}{\pi_{21}}\right)\right)\right) \\
= & N\left(\lambda, \frac{1}{n_{1}}\left(\frac{1}{\pi_{11}}+\frac{1}{\pi_{21}}\right)+\frac{1}{n_{2}}\right. \\
& \left.\times\left(\frac{1}{\pi_{21}}+\frac{1}{\pi_{21}}\right)\right) .
\end{aligned}
$$

A consistent estimator of the variance is provided by substituting the sample estimators and

$$
\hat{\boldsymbol{\sigma}}^{2}=\frac{1}{n_{11}}+\frac{1}{n_{21}}+\frac{1}{n_{12}}+\frac{1}{n_{22}} .
$$

